\title{
"Chase CRP", "Review patient": Improving the Quality of Weekend Medical Handover at a London Teaching Hospital
}

\author{
Aamir Saifuddin, Lucia Magee, Rachael Barrett \\ Charing Cross Hospital
}

\begin{abstract}
Clinical handover has been identified as a "major preventable cause of harm" by the Royal College of Physicians (RCP). Whilst working at a London teaching hospital from August 2013, we noted substandard weekend handover of medical patients. The existing pro forma was filled incompletely by day doctors so it was difficult for weekend colleagues to identify unwell patients, with inherent safety implications.

Furthermore, on-call medical staff noted that poor accessibility of vital information in patients' files was affecting acute clinical management. We audited the pro formas over a six week period $(n=83)$ and the Friday ward round (WR) entries for medical inpatients over two weekends $(n=84)$ against the RCP's handover guidance. The results showed poor documentation of several important details on the pro formas, for example, ceiling of care (4\%) and past medical history (PMH) (23\%). Problem lists were specified on $62 \%$ of the WR entries. We designed new handover pro formas and 'Friday WR sheets' to provide prompts for this information and used Medical Meetings and emails to explain the project's aims. Re-audit demonstrated significant improvement in all parameters; for instance, $\mathrm{PMH}$ increased to $52 \%$ on the pro formas. Only $10 \%$ of Friday WR entries used our sheet. However, when used, outcomes were much better, for example, problem list documentation increased to $100 \%$. In conclusion, our interventions improved the provision of crucial information needed to prioritise and manage patients over the weekend. Future work should further highlight the importance of safe handover to all doctors to induce a shift in culture and optimise patient care.
\end{abstract}

\section{Problem}

At Charing Cross Hospital, a tertiary referral centre in London in the United Kingdom, the weekend 'ward cover' medical team comprises a senior house officer (SHO) and a foundation year one doctor (FY1). They cover specialist respiratory and gastroenterology wards and three other wards that include elderly care, rheumatology and endocrinology patients, and those undergoing rehabilitation. This totals 120 patients. Anecdotal evidence from SHOs earlier in the year suggested that the quality of handover was poor for medical patients who needed specific weekend reviews or jobs. This seemed to impact greatly on the efficiency and effectiveness of the weekend team's work. There was also a suggestion that patient safety was put at risk for two reasons. First, the lack of detailed clinical information led to difficulties when the SHO had to delegate tasks to the FY1. Some patients, therefore, did not receive an appropriately senior review. Secondly, the written, non-verbal handover process did not highlight "unwell" patients who would need to be prioritised by the weekend team.

\section{Background}

Effective and comprehensive patient handover is crucial for the safe running of a hospital. The Royal College of Physicians (RCP) has identified handover as a "major preventable cause of harm." [1] This is especially important at the weekend. With the current working schedules of junior doctors, there may be as many as five distinct changes of medical personnel during the weekend. [1] This creates multiple opportunities for vital clinical information to be miscommunicated or omitted. To this end, the RCP has created a generic handover pro forma. [2]

Many novel ideas have been implemented in various hospitals to make the handover process more structured and safe. These have included new electronic systems and the use of mnemonics to remind staff of important pre-weekend tasks. [3-4] There is, however, large range in the sizes of hospitals, the types of patients that they deal with, the make-up of their on-call teams, and their electronic technology capabilities. Even though, therefore, the principles of effective handover are the same everywhere [1], in reality, specific local innovations are needed to address logistic and practical variations. At Charing Cross Hospital, the original handover system involved a weekend paper pro forma that had been devised two years ago. Junior doctors from each medical team would fill in details of each patient that needed to be reviewed and these sheets would be collected on Saturday morning by the weekend on-call team. The sheet's use, however, had not been formally audited and there were concerns about its effectiveness in prompting ward teams to provide comprehensive patient information. Nevertheless, written handovers are a very effective way of communicating clinical information. [5]

The aims of our work were:

1. To identify any shortcomings of the current weekend handover system from the perspective of junior doctors;

2. To audit the clinical data available for the weekend on-call team about the hospital's inpatients, many of whom will be unfamiliar to the them;

3. To implement appropriate changes based on our findings to improve patient safety over weekend; 


\section{BMJ Quality Improvement Reports}

4. To re-audit the outcome data and identify any further changes that need to be made.

\section{Baseline measurement}

Initial Surveys

We conducted written surveys amongst SHOs and spoke to registrars about the weekends. Six out of seven SHOs felt the current system was "unsafe". They noted that the limited patient information provided made it difficult for them to prioritise jobs, so unwell patients were often reviewed inappropriately late during the shift. Furthermore, the inefficiency caused by this sometimes resulted in the doctors simply not having enough time to see everyone on their lists. Interestingly, both Intensive Care and medical registrars independently explained how the quality of ward round entries is poor and this means it is very difficult when they need to review acutely unwell patients out-of-hours and make urgent decisions about care. One Intensive Care registrar noted that she often had to read through many pages of notes to find any documentation of a ceiling of care or a comprehensive 'problem list', which is clearly ineffective use of her time at a cardiac arrest situation.

In order to formally assess the effectiveness of the current handover system, we collected baseline measurements from the handover sheets and Friday ward round entries. These were audited against the domains on the RCP handover template. This serves as guidance for the clinical information that a doctor should know about an unfamiliar patient to be able to manage them appropriately out-of-hours. These domains included: patient name, date of birth (DoB), hospital number, ward, bed number, past medical history $(\mathrm{PMH})$, current issues, clear task, plan by team, task assignment (i.e. grade of doctor required for task), 'do not attempt resuscitation' (DNAR) status, ceiling of care, and discharge criteria.

We audited all of the weekend handover sheets during a six week period from October to November 2013. We also audited all Friday ward round entries for the hospital's four post-acute medical wards on two consecutive weeks in February 2014. Data for each was collected using a tick box style pro forma based on the aforementioned RCP guidance.

Handover sheets

We gathered data for 82 patients $(n=82)$. Name, hospital ID and ward were included over $80 \%$ of the time. Parameters documented poorly ( $<50 \%$ ) were: DoB, PMH, task assignment (i.e. FY1 or $\mathrm{SHO}$ ), DNAR status, ceiling of care, and discharge criteria, as per the data below and the attached graph.

Patient name: $100 \%$

DoB: $12 \%$

Hospital number: $88 \%$
Ward: $100 \%$

Bed number: $59 \%$

PMH: 23\%

Current issues: $67 \%$

Clear task: $80 \%$

Plan: $57 \%$

Task assignment: $30 \%$

DNAR status: $4 \%$

Ceiling of care: $4 \%$

Discharge criteria: $38 \%$

Several patient handovers simply included a patient name, hospital number and ward, and an instruction such as "check CRP", "review fluid status" or, simply, "review".

Friday ward round entries

We collected data from Friday ward round entries for 84 patients. The RCP criteria were again used to assess their quality. Furthermore, as junior doctors ourselves, this information was reviewed from the point of view of an on-call doctor, to gauge if there was sufficient detail to understand the key issues if we were called to see the patient urgently. There was good documentation of basic patient demographics. However, useful clinical information, such as primary diagnosis, problems list and ceiling of care, was documented less well:

Patient name: $93 \%$

DoB: $93 \%$

Hospital ID: $93 \%$

Primary diagnosis: $67 \%$

Problem list: $47 \%$

Ceiling of care: $2 \%$

Many ward round entries simply stated "Obs stable, continue as above", often for several days in a row. In many cases, there was no complete problem list for over one week, and for up to three weeks for some patients.

See supplementary file: ds4982.ppt - "Pre-intervention data and documents"

\section{Design}


After analysing the results, it was clear that completion of the handover pro forma and the detail provided in Friday ward round entries was often substandard. This is an obvious potential cause of patient harm as lack of relevant, readily available clinical information means that optimal management decisions cannot be made, both remotely and at the bedside. Handover instructions like "check fluid status" are extremely unhelpful when trying to prioritise jobs if they are not accompanied by other information - for instance, this could be for someone admitted recently with sepsis who may need to go to the Intensive Care Unit, or a patient on maintenance fluids who has a poor appetite. These both require different levels of prioritisation and, possibly, grades of doctor to manage them.

Similarly, ward round entries stating "Obs stable, continue" are unhelpful when the patient becomes acutely tachycardic or requires cardiopulmonary resuscitation (CPR). Someone then has to look through the notes to piece together the patient's medical history and find out the ceiling of care discussed with the patient and their family. We specifically sought to resolve these problems when devising our interventions.

We created a new weekend handover pro forma that was based on the RCP template (see attached material). This was in a grid format and was more prescriptive than the previous pro forma. There were headings for the specific information that would be required by the on-call team, to try to reduce the occasions where incomplete handovers are provided.

We also devised a Friday ward round sheet (see attached material). This contained areas for a complete acute problem list, relevant $\mathrm{PMH}$, ceiling of care, free space for the ward round entry itself, and specific weekend jobs and plans where applicable. The aim was for the ward round and key handover information to be documented simultaneously in the mornings, so there would be no additional work for the ward doctors later in the day. It was designed to serve as a prompt so that each patient had up to date information available for the weekend team. It was also hoped that the seniors conducting the ward round could help identify the key issues, which might improve the quality of the information.

\section{Strategy}

Handover pro forma

PDSA 1: The initial pro forma we designed contained separate boxes for each patient which were then subdivided in to sections such as demographics, current issues, and DNAR status. We hoped this would create space for more detailed handovers. However, only two sets of patient details could fit on the page. This was deemed both impractical and unsafe as the weekend doctors would have to assimilate tasks from multiple sheets of paper.

PDSA 2: We then devised the grid format so six patients would fit on each sheet. This revised pro forma was implemented for two weeks and feedback was sought. Even with this intuitive grid format, it was hard to identify the most unwell patients. We therefore added a "Sick?" column, which could simply be ticked if the patient needed to be prioritised on the Saturday morning.

PDSA 3: During the initial few weeks of its implementation, the width of the different columns was altered based on informal feedback. Following this, it was uploaded to the hospital intranet's shared drive and used weekly by all of the medical ward teams.

Friday ward round sheets

PDSA 1: The Friday WR sheets were trialled initially with one medical firm over three weeks. The juniors found the sheet useful for prompting them to generate up to date problems lists and to consider ceilings of care regularly. Following minor amendments to the formatting, the sheets were introduced to the other medical wards.

During the first two to three weeks, the sheet was used very frequently on most of the wards. The juniors, again, reported that they liked the prompt for a comprehensive problem list. We did worry initially that teams would simply see this as 'another piece of paper to fill in', so we tried hard to explain its benefits to the registrars and SHOs in person; they appreciated the reasons we had implemented it, especially as most of them had previously experienced problems with unsafe weekend handover.

\section{Post-measurement}

Following these PDSA cycles of active monitoring and changes, we left the interventions to run over several weeks before collecting further data to evaluate their efficacy.

\section{Handover pro forma}

We re-audited handover data from a total of 111 patients between March and May 2014. The previously well documented domains remained so. Those with poor outcomes pre-intervention nearly all reached above $50 \%$ compliance, as per the attached graphs. Furthermore, feedback suggested that the "sick?" box was a useful addition and reassured the day teams that their unwell patients would be reviewed appropriately by the on-call doctors.

\section{Friday WR sheets}

We audited ward round entries for two further weekends in April 2014 , comprising data for 88 patients. Only $10 \%$ of entries used our WR sheet. Junior doctors cited factors such as time constraints on a Friday and poor knowledge of their patients as reasons for this, though they appreciated that substandard WR entries could affect out-of-hours patient care. Where it was not used, there was no significant change in the outcomes compared to pre-intervention. This is despite providing information to junior and middle grade doctors about our initial findings in the Medical Meeting, along with follow-up emails and face to face discussions. When it was used, however, documentation was much better, including important domains like problem lists and ceilings of care. This is illustrated in the attached graphs. 
BMJ Quality Improvement Reports

See supplementary file: ds4981.ppt - "Interventions and PostIntervention data"

\section{Lessons and limitations}

Lesson one: Senior input helps direct change

Unfortunately not all consultants were available for the medical meeting where we presented our findings, and so were not fully aware of the project. We had hoped that those who were in attendance would encourage use of the Friday WR sheets, but not all of them round on a Friday. In one case a consultant did not wish to use the pro forma on their WR. By recruiting greater involvement at a senior level, promotion and uptake of our new Friday WR sheet might have been better.

Lesson two: Junior involvement facilitates action

Through our initial surveys, we gained formal and anecdotal feedback from junior and middle grade doctors that changes were needed to improve patient safety. In addition, we presented the preaudit data to our junior colleagues, indicating the rationale for a new system. The simple intervention of a different handover pro forma required minimal disruptions to the previous format. The Friday WR sheet, however, required active engagement and change in behaviour. We thought that provision of this pre-audit information alone would help direct change, but unfortunately this was not the case. We did, however, find that the juniors using the sheets the most were the ones with whom we had the most face-to-face contact. Unfortunately, we were unable to meet with every junior doctor personally, though investing time in this may be useful in future projects.

Limitations: There were only seven medical SHOs at the hospital, so only a limited amount of feedback could be obtained. Also, many of the FY1 and FY2 doctors rotated in and out of medicine during the year. Therefore, only a small number were able to directly compare our handover system with the pre-existing one and provide feedback.

\section{Conclusion}

In conclusion, a new weekend handover pro forma modelled on RCP guidance can improve the quality of information provided to the weekend team. This can help the on-call team to manage the weekend more effectively and safely. A Friday WR sheet can also improve the availability of thorough patient summaries in the notes in the event that someone needs to be reviewed unexpectedly over the weekend. Simply increasing awareness of the problems associated with substandard WR entries, however, did not help to improve their quality. Further work is needed to achieve this and to promote a change in culture regarding the importance of comprehensive handover so that patient safety can be optimised. We hope that we can carry this philosophy to other NHS Trusts that we work in and use our experiences to benefit other groups of doctors and patients.

\section{References}

1 https://www.rcplondon.ac.uk/sites/default/files/acute-caretoolkit-1-handover.pdf

2 https://www.rcplondon.ac.uk/sites/default/files/out-of-hourshandover_0.pdf

3 The F.R.I.D.A.Y.S. checklist - Preparing our patients for a safe weekend. Palmer E, Richardson E, Newcombe H, Borg C-M. BMJ Qual Improv Report 2013;2: doi:10.1136/bmjquality.u660.w502

4 Improving medical SHO weekend handover at a tertiary referral centre. Curtis O, Fisher R. BMJ Qual Improv Report 2013;2:doi:10.1136/bmjquality.u697.w971

5 An experimental comparison of handover methods. Bhabra G, Mackeith S, Monteiro P, Pothier DD. Ann R Coll Surg Engl. 2007 Apr;89(3):298-300

\section{Declaration of interests}

Nothing to declare.

\section{Acknowledgements}

The three authors contributed equally to the project overall and to the writing of the manuscript.

\section{Ethical approval}

Ethical approval was not required according to local policy because the project was for quality improvement and did not involve studies on human subjects. 